\title{
A transgenic mouse model for early prostate metastasis to lymph nodes
}

\author{
Hyun-Kyung Ko ${ }^{1}$, Shin Akakura1 ${ }^{1}$, Jennifer Peresie ${ }^{1}$, David W. Goodrich ${ }^{2}$, Barbara A. \\ Foster ${ }^{2}$, and Irwin H. Gelman ${ }^{1,}$, \\ ${ }^{1}$ Department of Cancer Genetics, Roswell Park Cancer Institute, Elm and Carlton Streets, \\ Buffalo, NY 14263 \\ 2Department of Pharmacology and Therapeutics, Roswell Park Cancer Institute, Elm and Carlton \\ Streets, Buffalo, NY 14263
}

\begin{abstract}
The emergence of recurrent, metastatic prostate cancer following the failure of androgendeprivation therapy represents the lethal phenotype of this disease. However, little is known regarding the genes and pathways that regulate this metastatic process, and moreover, it is unclear whether metastasis is an early or late event. The individual genetic loss of the metastasis suppressor, SSeCKS/Gravin/AKAP12, or $R b$, genes that are downregulated or deleted in human prostate cancer, results in prostatic hyperplasia. Here, we show that the combined loss of Akap12 and $R b$ results in prostatic intraepithelial neoplasia (PIN) that fails to progress to malignancy after 18 months. Strikingly, 83\% of mice with PIN lesions exhibited metastases to draining lymph nodes, marked by relatively differentiated tumor cells expressing markers of basal (p63, cytokeratin 14) and luminal (cytokeratin 8 and androgen receptor) epithelial cells, although none expressed the basal marker, cytokeratin 5. The finding that PIN lesions contain increased numbers of p63/AR-positive, cytokeratin 5-negative basal cells compared to WT or Akap12-/- prostate lobes suggests that these transitional cells may be the source of the LN metastases. Taken together, these data suggest that in the context of $R b$ loss, Akap12 suppresses the oncogenic proliferation and early metastatic spread of basal-luminal prostate tumor cells.
\end{abstract}

\section{Keywords}

SSeCKS/Gravin/Akap12; Rb; prostate cancer; metastasis; lymph node

\section{Introduction}

\begin{abstract}
Prostate cancer $(\mathrm{CaP})$ is the most commonly diagnosed non-cutaneous cancer and the second leading cause of cancer mortality in U.S. men (http://seer.cancer.gov/statfacts/html/ prost.html). During prostate carcinogenesis in humans, tumors progress from clinically undetectable precursor lesions, termed PIN (prostatic intraepithelial neoplasia), to locally invasive carcinomas often associated with metastatic disease to local lymph nodes and/or bones. Androgens act as pro-survival and proliferation factors in $\mathrm{CaP}$ cells such that chemical or surgical therapeutic castration to deplete serum androgen levels results in a rapid and in most cases, durable tumor regression. However, failure of androgen ablation
\end{abstract}

*corresponding author: 716-845-7681, Irwin.gelman@ roswellpark.org, 716-845-1698 fax.

The authors have no conflicts of interest in the current MS submission. 
therapy leads to so-called castration-recurrent (CR) $\mathrm{CaP}$, marked by more aggressive, metastatic disease that progresses in the presence of very low, intracrine androgen levels (1).

Genetic comparisons between primary site and $\mathrm{CaP}$ metastases within the same patient have been unable to resolve whether metastatic spread is an early or late event (2-4).

Comparative array-CGH analyses by Holcomb et al. (5) offer evidence of both possibilities: cases of strong relatedness between a patient's primary-site and metastatic lesions, suggesting late metastatic dissemination, versus cases in which there is more relatedness between metastases of different patients than their own primary-sites, suggesting early dissemination. Transgenic mouse $\mathrm{CaP}$ models have shown that at least two, and more typically, three genetic lesions are required to induce spontaneous metastasis (6). Yet, these models may be influenced by whether the oncogenic mutations are built into basal (7) or luminal prostate epithelial cells (8), or both (9). For example, building on evidence that $\mathrm{Rb}$ and p53 are often mutated or deleted in prostate cancer (10), a conditional Cre-loxP deletion of both $\mathrm{Rb}$ and $\mathrm{p} 53$ driven by a truncated probasin promoter (expressed in prostate and seminal vesicle epithelial cells) was produced (11). This model exhibits prostatic adenocarcinoma and lymph node metastases, whereas the loss of $\mathrm{Rb}$ alone causes prostatic hyperplasia (12).

SSeCKS/Gravin/AKAP12 ("Akap12") has been shown to function as a metastasis suppressor in prostate cancer, likely through its ability to scaffold key signaling and cytoskeletal proteins such as Src, protein kinase (PK)C, PKA, cyclin D and F-actin in a spatiotemporal manner (13). Akap12 re-expression suppressed macroscopic metastasis formation by inhibiting VEGF-mediated neovascularization at distal colonization sites (14). Akap12 is downregulated by Src-family kinases (15), known to be activated in CR-CaP progression $(16,17)$, and indeed, the forced re-expression of Akap12 suppresses Srcinduced oncogenic proliferation, invasiveness and cell motility in CaP cells by directly scaffolding Src from growth factor receptor and FAK complexes to lipid rafts, without altering Src's intrinsic kinase activity (18). Consistent with its function as a tumor suppressor in the prostate, there is significant Akap12 loss in $\mathrm{CaP}$ progression, due to either transcriptional downregulation or allelic deletion (6q24-25.3)(19). In addition, Akap12-null mice exhibit prostate hyperplasia marked by increased levels of cell senescence (20). Akap12-null mouse embryo fibroblasts (MEF) suffer an Rb-dependent premature senescence marked by multinucleation or polyploidy, suggesting a role for Akap12 in the regulation of cytokinesis and cell aging (21). The relative ease of isolating immortalized Akap12-null MEF in which components of the Rb pathway were downregulated suggested that the combined loss of Akap12 and Rb might be sufficient for oncogenic transformation. Here, we show that male Akap12-null mice with concomitant prostate-specific Rb deletion readily formed low- and high-grade PIN lesions by eight months of age. Unexpectedly, 83\% of these mice exhibit metastases in local draining lymph nodes, marked by relatively well differentiated tumor cells expressing markers of a transitional basal-luminal phenotype. Our data strongly suggest that $\mathrm{CaP}$ metastasis to the lymph node is an early event facilitated by the combined loss of Akap12 and $\mathrm{Rb}$ in the prostate.

\section{Materials and Methods}

\section{Generation of mutant mice}

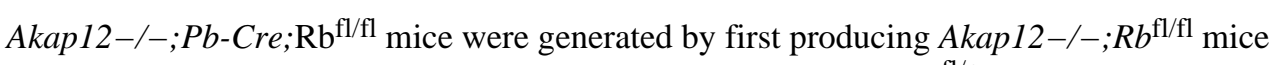
from crossing C57B/L6 Akap12-/- mice (20) with FVB/N;129 $R b^{\mathrm{fl} /+}$ mice (11), and then

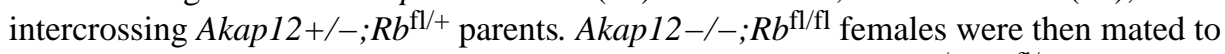
C57B/L6 xDBA2 Pb-Cre males (11), and the Akap12+/-;Pb-Cre ${ }^{+/-} ; \mathrm{Rb}^{\mathrm{fl} /++}$ males mated to Akap12-/-;Rb ${ }^{\mathrm{fl} / f \mathrm{l}}$ females over several generations to yield Akap12-/-;Pb-Cre; $\mathrm{Rb}^{\mathrm{fl} / \mathrm{fl}}$ mice. Note that the $\mathrm{Pb}$-Cre allele was only passaged through males in the intermediate 
lineages because of oocyte expression of the probasin promoter (23). All experiments were carried out in compliance with NIH guidelines and under the supervision of the RPCIIACUC.

\section{Genotyping}

$R b^{\mathrm{fl} / f 1}$ alleles were identified by PCR of tail-snip DNA using primers Rb18M3'

(5'GGCGTGTGCCATCAATG3') and Rb19EM5'(5'CTCAAGAGCTCAGACTCATGG3'); amplification of wild-type and floxed $R b$ gene sequences resulted in 235bp and 283bp DNA fragments, respectively, whereas Cre-excised alleles resulted in 260bp PCR products using primers Rb212M5' (5'CGAAAGGAAAGTCAGGGACATTGGG3') and Rb18M3'. Wildtype and Akap12-/- genotypes were identified as described previously (20).

\section{Immunohistochemistry (IHC) and immunofluorescence (IFA) analyses}

Tissues were fixed in 10\% buffered formalin (VWR, Radnor, PA) for 24h at RT prior to embedding in paraffin, and sectioning $(5 \mu \mathrm{m})$. Serial tissue sections each containing separated prostate lobes, seminal vesicle, lymph nodes (inguinal and pelvic), lung, liver and kidney were stained first with hematoxylin and eosin (H\&E) and then stained for Ki67 (Leica Biosystems, Buffalo Grove, IL, cat. \#NCL-Ki67p; dilution 1:500), TUNEL, cytokeratin (CK) 5 (Covance, Princeton, NJ, cat. \#PRB-160P; 1:2000), CK8 (Covance, cat. \#MMS-162P; 1:1000), CK14 (Abcam, Cambridge, MA, cat. \#Ab7800; 1:100), E-cadherin (Becton Dickinson Biosciences, San Jose, Ca, cat. \#610181; 1:500), androgen receptor (AR) (Upstate Biotechnology-Millipore, Billerica, MA, cat. \#06-680; 1:200), vimentin (Sigma, St. Louis, MO, cat. \#A5691; 1:400) or p63 (Santa Cruz Biotechnology, Santa Cruz, CA, cat. \#sc-8431; 1:1000) as described previously (20) using a DAKO Autostainer Plus system. Slides were dehydrated with graded alcohols and xylenes, then coverslipped, imaged and analyzed using an Aperio digital pathology system (Spectrum software v.11.0.0.725). Double IFA staining was performed on paraffin-embedded tissues with p63 (1:1000), CK5 (1:1000), and AR (Santa Cruz, cat. \#sc-816; 1:100). Secondary antibodies include 1:1000 dilutions of AlexaFluor 568- or 488-goat-anti-mouse IgG or goat-anti-rabbit IgG (Invitrogen-Molecular Probes, Grand Island, NY). Fluorescent images were acquired on a Nikon TE 2000-E inverted microscope (Melville, NY) using Metavue v.7.7.6.0 software (Molecular Devices, Sunnyvale, CA). Histological assessment was based on published guidelines (22). p63/CK5/AR IHC co-staining was quantified on an Aperio Imaging System. 200-300 total epithelial cell nuclei were counted in at least 6 independent prostate regions, and the number of $\mathrm{p} 63+/ \mathrm{CK} 5+, \mathrm{p} 63+/ \mathrm{CK}^{-}$, or $\mathrm{p} 63+/ \mathrm{AR}+$ cells were reported as a percentage of total epithelial cells in a lobe.

\section{RNA in situ hybridization of paraffin embedded tissue using DIG-labeled cRNA probes}

241-bp cDNA templates for antisense or sense RNA probes were generated by PCR amplification $\left(95^{\circ} \mathrm{C} / 3 \mathrm{~min} ; 37 \mathrm{cycles}\right.$ of $\left.95^{\circ} \mathrm{C} / 30 \mathrm{sec}, 55^{\circ} \mathrm{C} / 30 \mathrm{sec}, 72^{\circ} \mathrm{C} / 30 \mathrm{sec}\right)$ of pBabehygro-Cre plasmid DNA (gift of Eugene Kandel, RPCI) with the following primer pairs containing T7 RNA polymerase promoter sites (underlined):

Sense: forward, $5^{\prime}-$ TAATACGACTCACTATAGGGGCATTTCTGGGGATTGCTTA- $3^{\prime}$ reverse, 5'-CCCGGCAAAACAGGTAGTTA-3

Anti-sense: forward, 5'-GCATTTCTGGGGATTGCTTA-3' reverse, 5'-TAATACGACTCACTATAGGGCCCGGCAAAACAGGTAGTTA-3'

Digoxigenin-UTP-labeled antisense and sense RNA probes were generated by in vitro transcription using a DIG RNA Labeling Kit (Roche Applied Science, cat. \#11175025910) 
according to the manufacturer's instructions. The concentration of RNA probes was measured using a Thermo Scientific-Nanodrop and then adjusted with DEPC-treated water to $500 \mathrm{ng} / \mu \mathrm{l}$. In situ hybridization was carried out as follows: $5 \mu \mathrm{m}$ sections of the formalinfixed paraffin-embedded mouse prostate tissue was deparaffinized in three changes of xylene for $7 \mathrm{~min}$ each, washed twice in $100 \%$ ethanol for $5 \mathrm{~min}$, followed by rehydration in a graded series of ethanols $(95 \%, 70 \%$, and 50\%, 5 min each) and two washes in DEPC water for $3 \mathrm{~min}$. Endogenous peroxidase activity was blocked with $3 \%$ hydrogen peroxide in DEPC water for $15 \mathrm{~min}$ and rinsed twice with DEPC water. The sections were then incubated at $37^{\circ} \mathrm{C}$ for $30 \mathrm{~min}$ in Tris-buffered saline (TBS: $50 \mathrm{mM}$ Tris, $\mathrm{pH} 7.5,180 \mathrm{mM} \mathrm{NaCl}$ ) containing proteinase $\mathrm{K}(20 \mu \mathrm{g} / \mathrm{ml})$. After two washes in TBST (TBS plus $1 \%$ Triton X-100) for $3 \mathrm{~min}$ each, the sections were pre-hybridized for $1 \mathrm{~h}$ at $55^{\circ} \mathrm{C}$ in with $100 \mu \mathrm{l}$ hybridization buffer (Chemicon, Temecula, CA, cat. \#S4040). DIG labeled sense or anti-sense probes were diluted to $1 \mathrm{ng} / \mu \mathrm{l}$ in $150 \mu \mathrm{l}$ of hybridization buffer, denatured at $65^{\circ} \mathrm{C}$ for $2 \mathrm{~min}$ and put on ice. Tissue sections were covered with the denatured RNA probes and incubated for $16-18 \mathrm{~h}$ at $55^{\circ} \mathrm{C}$. Unhybridized probe was removed by washing in $2 \mathrm{X}$ pre-warmed SSC (300 $\mathrm{mM} \mathrm{NaCl}, 30 \mathrm{mM}$ trisodium citrate, $\mathrm{pH} 7.0$ ) at $45^{\circ} \mathrm{C}$ for $10 \mathrm{~min}$ and $\mathrm{RT}$ for $5 \mathrm{~min}$, and subsequently incubated for $30 \mathrm{~min}$ at $37^{\circ} \mathrm{C}$ with $250 \mu \mathrm{l}$ of RNAse Cocktail (InvitrogenAmbion, cat. \# AM2286) diluted 1:30 in 2X SSC. The slides were washed twice at highstringency in pre-warmed $50 \%$ formamide in $2 \mathrm{X} \mathrm{SSC}$ for $30 \mathrm{~min}$ at $55^{\circ} \mathrm{C}$, once with prewarmed $0.08 \mathrm{X} \mathrm{SSC}$ for $20 \mathrm{~min}$ at $55^{\circ} \mathrm{C}$, and then rinsed twice with TBST. Probe hybridization was detected using a DAKO autostainer, as follows: tissue sections were blocked with Super Block (Thermo-Pierce, cat. \#37535) for $1 \mathrm{hr}$, incubated for $30 \mathrm{~min}$ with anti-DIG-HRP conjugate (Roche, \#11207733910; 1:100) in Super Block, three washes with TBST for 5 min each, and then for 15 min with biotinylated rabbit anti-sheep Ig (Vector Labs, Burlingame, CA, cat. \#BA-6000; 1:1000). Signal enhancement was facilitated by 30 min incubation with $\mathrm{ABC}$ reagent (Vector, cat. \#PK 6100), 5 min incubation with DAB substrate (Dako-Agilent, Carpinteria, CA, cat. \#K3467) and counterstaining with modified Harris hematoxylin (Thermo-Richard-Allan Scientific, Waltham, MA, cat. \#72704) for 20 sec. The slides were dehydrated with graded alcohols and then xylenes, coverslipped and digitally analyzed using an Aperio system.

\section{Results}

\section{Combined deletion of $A k a p 12$ and $R b$ leads to PIN within 6 months}

To assess a possible cooperation between Akap12 and $\mathrm{Rb}$ in prostate carcinogenesis, Akap12-null mice were crossed with $\mathrm{Pb}-\mathrm{Cre} ; \mathrm{Rb}^{\mathrm{fl} / f \mathrm{l}}$ (11), resulting in Akap12-/-;Pb-

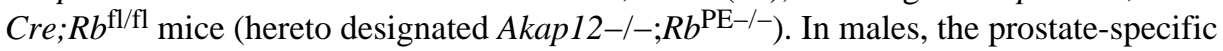
composite rat probasin promoter, $\mathrm{Pb} 4$, is expressed in basal and luminal prostate epithelium in all prostatic lobes (23). Prostate-specific deletion of the $R b$ exon 19 in all lobes was confirmed by PCR (Supplementary fig. S1A) in 3 week-old mice. Importantly, no Cremediated recombination was detected in other organs such as the inguinal lymph nodes and kidney (Supplementary fig. S1B).

As reported previously, Akap12-null mice exhibit prostatic hyperplasia starting at 4 months of age (20) (Fig. 1A); similar effects were produced in $\mathrm{Pb}-\mathrm{Cre} ; \mathrm{R} b^{\mathrm{fl} / \mathrm{fl}}$ mice $(11,12)$. Yearold Akap12-null mice showed focal dysplasia in fewer than $30 \%$ of cases, and one case of low-grade prostatic intraepithelial neoplasia (LGPIN) was detected in an 18 month-old (Fig. 1B). In contrast, Akap12-/-;Rb $b^{\mathrm{PE}-/-}$ developed LGPIN in all of prostate lobes starting at 5 months (Fig. 1B) marked by a focal loss of columnar polarity containing cells with slightly hyperchromatic, elongated and irregular nuclei (Fig. 1A). 29\% of Akap12-/-;Rb $b^{\mathrm{PE}-/-}$ mice also exhibited high grade (HG) PIN (Table 1) between 6-13 months of age marked by foci 
highly atypical cells with severe nuclear pleomorphism and hyperchromasia (Fig. 1A). DP and LP lobes exhibited a more severe phenotype, including HGPIN, than VP or AP lobes.

In order to better classify the neoplastic phenotype of the prostate lesions in Akap12-I $-; R b^{\mathrm{PE}-/-}$ mice according to the criteria set forth by Pienta et al. (22), DP lobes from WT, Akap12-/-, or Akap12-/-;Rb $b^{\mathrm{PE}-/-}$ mice were analyzed by immunohistochemistry (IHC) for progression biomarkers. Compared to Akap12-/- prostates, Akap12-/-;Rb $b^{\mathrm{PE}-/-}$ prostates displayed increased numbers of Ki67- and p63-positive cells, increased nuclear AR staining levels and increased cytoplasmic CK8 levels (Fig. 2, panels A, C, D and F). AR expression is E2F1-inducible, and thus, its upregulation would be expected following the loss of $R b$ (24). Total E-cadherin staining was increased although heterogeneous, and significantly, there was little of the normal cell-cell demarcation pattern still retained in the hyperplastic Akap12-/- lesions, owing to the loss of polar epithelial organization in the PIN lesions (Fig. 2, panel B). Unexpectedly, the increased number of p63-positive cells in Akap12-l-;Rb $\mathrm{PE}^{\mathrm{PE}-}$ lesions did not correlate with a similar increase in positivity for CK5 (Fig. 2, panel E), another basal epithelial cell marker. The relative slow growth of these PIN lesions even in the presence of increased apparent proliferation markers was likely explained by a concomitant increase in apoptosis, as measured by TUNEL assay (Supplementary figs. S2A $\& \mathrm{~B})$. It is noteworthy that the $A k a p 12-/-; R b^{\mathrm{PE}-/-}$ lesions displayed increased stromal layers as well as infiltrates of possible inflammatory cells (Fig. 1A) that were strongly positive for Ki67 and AR staining (Fig. 2, panels A and C).

\section{Basal cell proliferation in Akap12-I-;R $b^{\mathrm{PE}-/-}$ PIN lesions}

A hallmark of many transgenic mouse models of PIN or prostatic adenocarcinoma is a concomitant loss of basal cells amidst proliferating epithelial cells expressing luminal markers such as CK8 or 18 (22). However, there is evidence that prostate cancer can arise from basal epithelial cells that transition to a luminal phenotype including induction of AR and CK8 expression $(7,9)$. We followed up on the observation of increased numbers of p63-

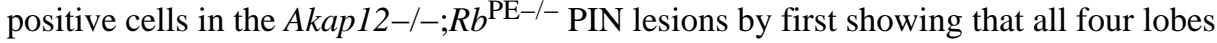
displayed significantly increased numbers of basal cells, especially in the DP and AP, compared to WT or Akap12-/- mice (Fig. 3A). Moreover, the normally flat cell morphology of a basal cell layer is replaced by apolar cells with enlarged, atypically-shaped nuclei containing segmented chromatin (Fig. 3B). Double staining for p63 and CK5 showed that WT and Akap12-/- basal cells had fully concordant co-expression of both markers, whereas there was a $35 \%$ decrease p63-positive cells in the Akap12-I-;Rb $b^{\mathrm{PE}-/-}$ lesions costaining with CK5 compared to controls (Fig. 3C, left panels; Fig. 3D; Supplementary fig. S3). Additionally, whereas nuclear androgen receptor (AR) staining was mainly in WT or Akap12-/- luminal cells (Fig. 3C, right panels; Supplementary fig. S3), many Akap12-/ $-; R b^{\mathrm{PE}-/-}$ cells displayed combined p63- and AR-positivity. Taken together, these data suggest that Akap12-/-;Rb $b^{\mathrm{PE}-/-}$ PIN lesions contain both proliferating luminal as well as transitional basal-luminal epithelial cells.

\section{Loss of Akap12 and Rb results in significant prostate metastasis to the lymph node}

None of the Akap12-I-;Rb $b^{\mathrm{PE}-/-}$ mice aged to 24 months showed evidence of adenocarcinoma, indicating that the combined loss of $A k a p 12$ and $R b$ was only partially oncogenic in regards to the primary prostate site. However, consistent with Akap12 functioning as a metastasis suppressor in prostate cancer cell lines $(14,18,25)$, and with the increased levels of $A K A P 12$ promoter hypermethylation in human prostate cancer lesions with Gleason grades $8-10$ (26), 83\% of the Akap12-/-;Rb $b^{\mathrm{PE}-/-}$ mice that had PIN lesions also displayed metastases in their pelvic or inguinal lymph nodes (LN)(Fig. 4A; Table 2). In contrast, none of the WT or Akap12-/- mice had such lesions. Moreover, this frequency of metastasis is significantly higher than in mice with combined deletions of $R b$ and p53 in the 
prostate $(83 \%$ vs. $68 \%+/-8)(11)$. All mice with LN metastases had foci of primary site HGPIN, many of which showed clear evidence of localized invasiveness, an example of which is shown in Supplementary fig. S4A. Notably, macroscopic metastases were not detected in other peripheral organs or non-pelvic LN, and moreover, the pelvic LN lesions (Fig. 4A) were detected as early as one month following the earliest appearance of PIN lesions (Fig. 1B). We cannot rule out the possibility that other sites contain micrometastases. However, to address whether the increased metastasis in Akap 12-I-;Rb $b^{\mathrm{PE}-/-}$ mice correlates with changes in circulating tumor cells (CTC), peripheral blood samples obtained by cardiac puncture from Akap12-/-;R $b^{\mathrm{PE}-/-}$, Akap12-/- or $R b^{\mathrm{PE}-/-}$ mice (6 mice/group) were analyzed by sensitive PCR for CTCs, either for the floxed form of $R b$ or for the $n e o^{\mathrm{R}}$ cassette (in Akap12-/- mice). Although this assay could readily detect 1-CTC in $10^{5}-10^{6}$ buffy coat cells (Supplementary fig. S5A), no increases in CTCs were found in Akap12-/ $-; R b^{\mathrm{PE}-/-}$ compared to Akap12-l- mice (Supplementary fig. S5B). This strongly suggests

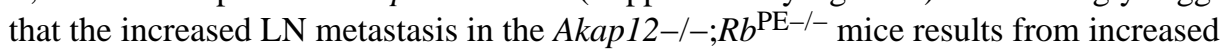
localized trafficking, possibly through the known ability of SSeCKS/Akap12 to regulate chemotaxis $(25,27)$. Another characteristic of the metastases is that these lesions are primarily within the LN sinusoids, with little or no evidence of invasion to the lymphoid follicles (Fig. 4B). This suggests that the disseminating cells are a subset of primary-site PIN cells that have gained trafficking potential yet have failed to re-differentiate to establish a mature metastatic focus.

The LN metastases contained tumor cells with highly epithelioid morphologies, lacking mesenchymal morphologies typical of aggressiveness. Moreover, they expressed both basal and luminal epithelial markers: strong staining for $\mathrm{p} 63$ (96\% of mice with positive staining), CK8 and 14 (78\% and 67\% positivity, respectively), E-cadherin (82\% positivity) and vimentin (60\% positivity) but not for CK5 (0\% positivity)(Fig. 4B; Supplementary fig. S6). It is noteworthy that the same CK5 antibody stained basal epithelial cells in prostate lobes (Fig. 2E) on the same slide as the LN samples, ruling out the possibility of a technical artifact. Staining for nuclear AR and E-cadherin was heterogeneous amongst cells within LN lesions (Fig. 4B), although there was a significant increase in TUNEL-positive apoptotic cells compared to controls LNs (Supplementary fig. 2C). Fewer than 5\% of the LN lesions homogeneously expressed the neuroendocrine marker, synaptophysin (Supplementary fig. S4B), and taken with the expression of cytokeratins in the remaining 95\%, this indicates that the vast majority were likely adenocarcinoma precursors. We excluded the possibility that the LN lesions were CD68-positive histiocytes (Supplementary fig. S6), i.e.- cells that might be recruited in cases of chronic inflammation.

Similar to the mild oncogenic penetrance of the primary PIN lesions, the LN metastases did not grow aggressively although the inguinal LNs of 10 month-old Akap12-I-;Rb $b^{\mathrm{PE}-/-}$ mice typically weighed 3-4 times more than Akap12-/- controls. Notably, foci could be detected within Akap 12-I-;R $b^{\mathrm{PE}-/-}$ LNs that contained cells with severe nuclear pleomorphism and abnormal nucleus to cytoplasm ratios (Fig. 4B, inset arrow) typical of HGPIN or carcinoma. PCR analysis of DNA isolated from laser microdissected LN or prostate tumor tissue indicated that the LN metastases were of prostatic origin in that they, but not tail DNA, encode Cre-mediated deletions of the $R b^{\mathrm{fl} / f \mathrm{l}}$ locus (Fig. 4C). Importantly, LN DNA from 3 week-old Akap12-I-;R $b^{\mathrm{PE}-/-}$ newborns were negative for the $R b^{\mathrm{fl} / \mathrm{fl}}$ locus deletion (Supplementary fig. S1B), strongly suggesting that the $R b$-deleted cells in the adult LN originated from prostates.

In order to strengthen the notion that that most, if not all, the LN lesion cells were of prostate origin, we performed RNA in situ hybridization using single-stranded Cre probes. The Cre probe showed no hybridization signals in primary VP lobes or LN from WT, $R b^{\mathrm{PE}-/-}$ or Akap12-/- mice (Fig. 4D; Supplementary fig. S7), whereas it induced strong 
cytoplasmic signals in both the PIN and LN lesions from Akap12-/-;Rb $b^{\mathrm{PE}-/-}$ mice (Fig. 4D). Moreover, young Akap 12-/-;Rb $b^{\mathrm{PE}-/-}$ mice ( $<4 \mathrm{mo}$.), which show no evidence primary-site PIN disease (Fig. 1B), showed no Cre expression in LN (Supplementary fig. S7). These data strongly suggest that the loss of Akap12 can potentiate an early metastatic colonization of $R b$-negative prostate cells to local LNs, although oncogenic progression in the prostate and LN is limited to non-malignant HGPIN.

\section{DISCUSSION}

Building on our observation that the loss of Akap12 induces Rb-dependent senescence associated with the hyperplasia of luminal prostate epithelial cells (21), we now show that the crossing a prostate-specific $R b$ loss into an Akap12-null background induces PIN lesions that do not readily progress to adenocarcinomas. Indeed, whereas spontaneous death was rare even in 16-20 month-old Akap 12-I-;Rb ${ }^{\mathrm{PE}-/-}$ mice, the median survival for the combined prostate-specific loss of p53 and Rb was only 200 days (11). Although the prostate lesions in Akap12-I-;Rb $b^{\mathrm{PE}-/}$ mice display increased markers of proliferation, such as Ki67, it is likely that a concomitant increase in apoptosis in all four prostate lobes (as measured by TUNEL staining) antagonizes further progression to malignancy. This suggests that the combined loss of $\mathrm{Rb}$ and Akap12 fails to fully defeat all senescence pathways. Examples of parallel, tertiary senescence control mediators include p21, which mediates senescence of PC-3 CaP cells following activation of the androgen receptor (28), or SMAD4, which facilitates CaP malignancy in the background of PTEN loss (29).

Given the lack of malignant progression of the primary prostate lesions in Akap12-/ $-; R b^{\mathrm{PE}-/-}$ mice, the high incidence of $\mathrm{LN}$ metastasis was notable and suggests that prostate cancer metastasis to the LN can be an early event. The LN lesions, all of which derived from mice with focal HG-PIN, contained cells with epithelioid, non-aggressive morphologies and expressed both basal and luminal prostate epithelial markers, such as CK14, p63, CK8, Ecadherin and AR, but not the basal marker, CK5. This correlated in the primary-site PIN lesions with a significant increase in total $\mathrm{p} 63^{+}$cells yet a relative decrease in coincident CK5 co-staining, The $\mathrm{p}^{+} 3^{+} \mathrm{CK}^{-}$cells in the primary PIN lesion lack typical basal cell morphologies and polarization, and the increase in this population correlates with increased nuclear AR expression. Although further experiments are required, it is interesting to speculate that the $\mathrm{LN}$ metastases arise from basal cell populations that are transitioning to more luminal phenotypes. Interestingly, over $50 \%$ of human prostate cancer cases with minimal primary site disease show evidence of PSA-positivity in the bone marrow (2) or disseminated tumor cells in local lymph nodes (30), strongly suggesting early metastatic dissemination. Given that the majority of initial $\mathrm{CaP}$ metastases in the clinical setting are found in local draining $\mathrm{LN}$ and bones

In regards to the CK5-negative lesions in our model's PIN and LN lesions, although human CK5-positive basal prostate epithelial cells can serve as progenitors of luminal prostate cancers $(7,31)$, and CK5/18-positive transitional cells have been described in human $\mathrm{CaP}$ (32), small populations of CK5-negative basal cells have been described (33) which our genetic alterations may have selected. Moreover, CK14-expressing prostatic basal cells can serve as $\mathrm{CaP}$ progenitors in transgenic models irrespective of their ability to express CK5 (9).

Previous data from our group indicated that Akap12 can suppress metastatic motility, chemotaxis and invasiveness by inhibiting PKC-mediated pathways through direct scaffolding of PKC isoforms (25) and by physically scaffolding Src away from FAK adhesion complexes, thereby attenuating MEK/ERK pathways controlling oncogenic motility (18). Thus, the ability of Akap12 to attenuate Src-induced oncogenic pathways (18, 
$25,34,35)$ likely contributes to its metastasis-suppressing activity, especially given the renewed focus on the role of Src-family kinases in mediating several parameters of metastatic and castration-recurrent prostate cancer $(36,37)$.

Our findings are consistent with a role for Akap12 in suppressing metastatic progression such that loss of Akap12 in specific tissues, such as the prostate epithelium, results in a metastasis-prone condition (Fig. 4E). Indeed, of the 37 cases of metastatic CaP from Taylor et al. (38) analyzed in the cbio website (http://www.cbioportal.org/public-portal/), the 11 cases which displayed AKAP12 downregulation correlated with a more rapid appearance of metastases compared to the 26 cases that showed no changes in AKAP12 expression levels (Fig. 4F). Taken together with our findings, these data argue strongly for a role for AKAP12 in suppressing specific parameters of metastatic growth of prostate cancer.

\section{Supplementary Material}

Refer to Web version on PubMed Central for supplementary material.

\section{Acknowledgments}

We thank Bryan Gillard and Ellen Karasik for excellent technical assistance, and Eugene Kandel for sharing reagents and Gissou Azabdaftari for advice on pathologic analyses. This work was supported by NIH grants CA70292 (D.G.), CA94108 and CA116430 (I.H.G.), and DoD grants PC074228 and PC101210 (I.H.G.), and in part, through NCI Comprehensive Cancer funds (P30-CA016056).

\section{Reference List}

1. Jin JK, Dayyani F, Gallick GE. Steps in prostate cancer progression that lead to bone metastasis. Int J Cancer. 2011; 128:2545-61. [PubMed: 21365645]

2. Melchior SW, Corey E, Ellis WJ, Ross AA, Layton TJ, Oswin MM, et al. Early tumor cell dissemination in patients with clinically localized carcinoma of the prostate. Clin Cancer Res. 1997; 3:249-56. [PubMed: 9815680]

3. Lapointe J, Li C, Giacomini CP, Salari K, Huang S, Wang P, et al. Genomic profiling reveals alternative genetic pathways of prostate tumorigenesis. Cancer Res. 2007; 67:8504-10. [PubMed: 17875689]

4. Holcomb IN, Grove DI, Kinnunen M, Friedman CL, Gallaher IS, Morgan TM, et al. Genomic alterations indicate tumor origin and varied metastatic potential of disseminated cells from prostate cancer patients. Cancer Res. 2008; 68:5599-608. [PubMed: 18632612]

5. Holcomb IN, Young JM, Coleman IM, Salari K, Grove DI, Hsu L, et al. Comparative analyses of chromosome alterations in soft-tissue metastases within and across patients with castration-resistant prostate cancer. Cancer Res. 2009; 69:7793-802. [PubMed: 19773449]

6. Hensley PJ, Kyprianou N. Modeling Prostate Cancer in Mice: Limitations and Opportunities. J Androl. 2011; 33:133-44. [PubMed: 21680808]

7. Goldstein AS, Huang J, Guo C, Garraway IP, Witte ON. Identification of a cell of origin for human prostate cancer. Science. 2010; 329:568-71. [PubMed: 20671189]

8. Wang X, Kruithof-de JM, Economides KD, Walker D, Yu H, Halili MV, et al. A luminal epithelial stem cell that is a cell of origin for prostate cancer. Nature. 2009; 461:495-500. [PubMed: 19741607]

9. Choi N, Zhang B, Zhang L, Ittmann M, Xin L. Adult Murine Prostate Basal and Luminal Cells Are Self-Sustained Lineages that Can Both Serve as Targets for Prostate Cancer Initiation. Cancer Cell. 2012; 21:253-65. [PubMed: 22340597]

10. MacGrogan D, Bookstein R. Tumour suppressor genes in prostate cancer. Semin Cancer Biol. 1997; 8:11-9. [PubMed: 9299577]

11. Zhou Z, Flesken-Nikitin A, Corney DC, Wang W, Goodrich DW, Roy-Burman P, et al. Synergy of p53 and Rb deficiency in a conditional mouse model for metastatic prostate cancer. Cancer Res. 2006; 66:7889-98. [PubMed: 16912162] 
12. Maddison LA, Sutherland BW, Barrios RJ, Greenberg NM. Conditional deletion of Rb causes early stage prostate cancer. Cancer Res. 2004; 64:6018-25. [PubMed: 15342382]

13. Gelman IH. Emerging Roles for SSeCKS/Gravin/AKAP12 in the Control of Cell Proliferation, Cancer Malignancy, and Barriergenesis. Genes \& Cancer. 2010; 1:1147-56. [PubMed: 21779438]

14. Su B, Zheng Q, Vaughan MM, Bu Y, Gelman IH. SSeCKS metastasis-suppressing activity in MatLyLu prostate cancer cells correlates with VEGF inhibition. Cancer Res. 2006; 66:5599-607. [PubMed: 16740695]

15. Lin X, Tombler E, Nelson PJ, Ross M, Gelman IH. A novel src- and ras-suppressed protein kinase C substrate associated with cytoskeletal architecture. J Biol Chem. 1996; 271:28430-8. [PubMed: 8910468]

16. Chang YM, Kung HJ, Evans CP. Nonreceptor tyrosine kinases in prostate cancer. Neoplasia. 2007; 9:90-100. [PubMed: 17357254]

17. Saad F. Src as a therapeutic target in men with prostate cancer and bone metastases. BJU Int. 2009; 103:434-40. [PubMed: 19154462]

18. Su B, Gao L, Meng F, Guo LW, Rothschild J, Gelman IH. Adhesion-mediated cytoskeletal remodeling is controlled by the direct scaffolding of Src from FAK complexes to lipid rafts by SSeCKS/AKAP12. Oncogene. 2012

19. Xia W, Unger P, Miller L, Nelson J, Gelman IH. The Src-suppressed C kinase substrate, SSeCKS, is a potential metastasis inhibitor in prostate cancer. Cancer Res. 2001; 61:5644-51. [PubMed: 11454719]

20. Akakura S, Huang C, Nelson PJ, Foster B, Gelman IH. Loss of the SSeCKS/Gravin/AKAP12 gene results in prostatic hyperplasia. Cancer Res. 2008; 68:5096-103. [PubMed: 18593908]

21. Akakura S, Nochajski P, Gao L, Sotomayor P, Matsui S, Gelman IH. Rb-dependent cellular senescence, multinucleation and susceptibility to oncogenic transformation through PKC scaffolding by SSeCKS/AKAP12. Cell Cycle. 2010; 9:4656-65. [PubMed: 21099353]

22. Pienta KJ, Abate-Shen C, Agus DB, Attar RM, Chung LW, Greenberg NM, et al. The current state of preclinical prostate cancer animal models. Prostate. 2008; 68:629-39. [PubMed: 18213636]

23. Wu X, Wu J, Huang J, Powell WC, Zhang J, Matusik RJ, et al. Generation of a prostate epithelial cell-specific Cre transgenic mouse model for tissue-specific gene ablation. Mech Dev. 2001; 101:61-9. [PubMed: 11231059]

24. Aparicio A, Den RB, Knudsen KE. Time to stratify? The retinoblastoma protein in castrateresistant prostate cancer. Nat Rev Urol. 2011; 8:562-8. [PubMed: 21811228]

25. Su B, Bu Y, Engelberg D, Gelman IH. SSeCKS/Gravin/AKAP12 inhibits cancer cell invasiveness and chemotaxis by suppressing a PKC-RAF/MEK/ERK pathway. J Biol Chem. 2010; 285:457886. [PubMed: 20018890]

26. Liu W, Gong J, Hu J, Hu T, Sun Y, Du J, et al. Quantitative Assessment of AKAP12 Promoter Methylation in Human Prostate Cancer Using Methylation-sensitive High-resolution Melting: Correlation With Gleason Score. Urology. 2011; 77:1006, e1-7. [PubMed: 21310466]

27. Su B, Gao L, Meng F, Guo LW, Rothschild J, Gelman IH. Adhesion-mediated cytoskeletal remodeling is controlled by the direct scaffolding of Src from FAK complexes to lipid rafts by SSeCKS/AKAP12. Oncogene. 2013; 32:2016-26. [PubMed: 22710722]

28. Mirochnik Y, Veliceasa D, Williams L, Maxwell K, Yemelyanov A, Budunova I, et al. Androgen receptor drives cellular senescence. PLoS ONE. 2012; 7:e31052. [PubMed: 22403609]

29. Ding Z, Wu CJ, Chu GC, Xiao Y, Ho D, Zhang J, et al. SMAD4-dependent barrier constrains prostate cancer growth and metastatic progression. Nature. 2011; 470:269-73. [PubMed: 21289624]

30. Schilling D, Hennenlotter J, Sotlar K, Kuehs U, Senger E, Nagele U, et al. Quantification of tumor cell burden by analysis of single cell lymph node disaggregates in metastatic prostate cancer. Prostate. 2010; 70:1110-8. [PubMed: 20503396]

31. Tokar EJ, Ancrile BB, Cunha GR, Webber MM. Stem/progenitor and intermediate cell types and the origin of human prostate cancer. Differentiation. 2005; 73:463-73. [PubMed: 16351690]

32. van Leenders GJ, Aalders TW, Hulsbergen-van de Kaa CA, Ruiter DJ, Schalken JA. Expression of basal cell keratins in human prostate cancer metastases and cell lines. J Pathol. 2001; 195:563-70. [PubMed: 11745692] 
33. Man YG, Zhao C, Chen X. A subset of prostate basal cells lacks the expression of corresponding phenotypic markers. Pathol Res Pract. 2006; 202:651-62. [PubMed: 16842934]

34. Gelman IH, Gao L. The SSeCKS/Gravin/AKAP12 Metastasis Suppressor Inhibits Podosome Formation Via RhoA- and Cdc42-Dependent Pathways. Mol Cancer Res. 2006; 4:151-8. [PubMed: 16547152]

35. Lin X, Gelman IH. Re-expression of the major protein kinase C substrate, SSeCKS, suppresses v$s r c$-induced morphological transformation and tumorigenesis. Cancer Res. 1997; 57:2304-12. [PubMed: 9187136]

36. Gallick GE, Corn PG, Zurita AJ, Lin SH. Small-molecule protein tyrosine kinase inhibitors for the treatment of metastatic prostate cancer. Future Med Chem. 2012; 4:107-19. [PubMed: 22168167]

37. George D, Moul JW. Emerging treatment options for patients with castration-resistant prostate cancer. Prostate. 2012; 72:338-49. [PubMed: 21748753]

38. Taylor BS, Schultz N, Hieronymus H, Gopalan A, Xiao Y, Carver BS, et al. Integrative genomic profiling of human prostate cancer. Cancer Cell. 2010; 18:11-22. [PubMed: 20579941]

39. Lapointe J, Li C, Higgins JP, van de RM, Bair E, Montgomery K, et al. Gene expression profiling identifies clinically relevant subtypes of prostate cancer. Proc Natl Acad Sci U S A. 2004; 101:811-6. [PubMed: 14711987] 


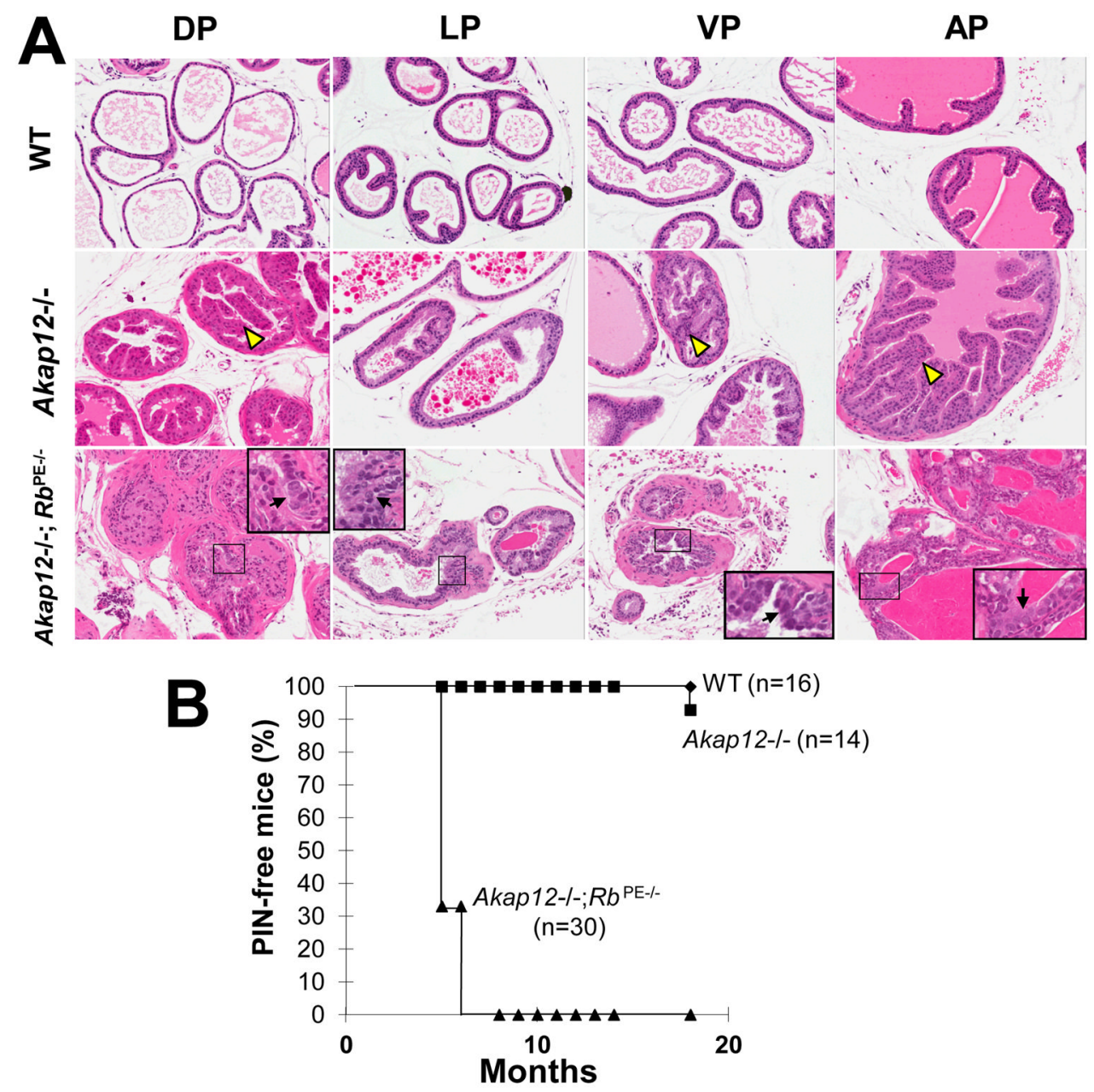

Fig. 1. The combined loss of Akap12 and Rb results in PIN lesions

A. H\&E staining of formalin-fixed paraffin sections of prostatic lobes from 8 month-old WT, Akap 12-/-, and Akap 12-/-;R $b^{P E-/-}$ mice. The hyperplasia of luminal epithelial cells found in Akap12-/- mice (yellow arrowhead) was replaced by PIN (arrow) in Akap12-/ $-; R b^{\mathrm{PE}-l-}$ mice. Insets show nuclear atypia with prominent nucleoli. B. Graphic analysis of PIN formation in WT, Akap12-/-, and Akap12-/-;Rb $b^{P E-/-}$ mice over time, based on mouse numbers (n) per genotype. 

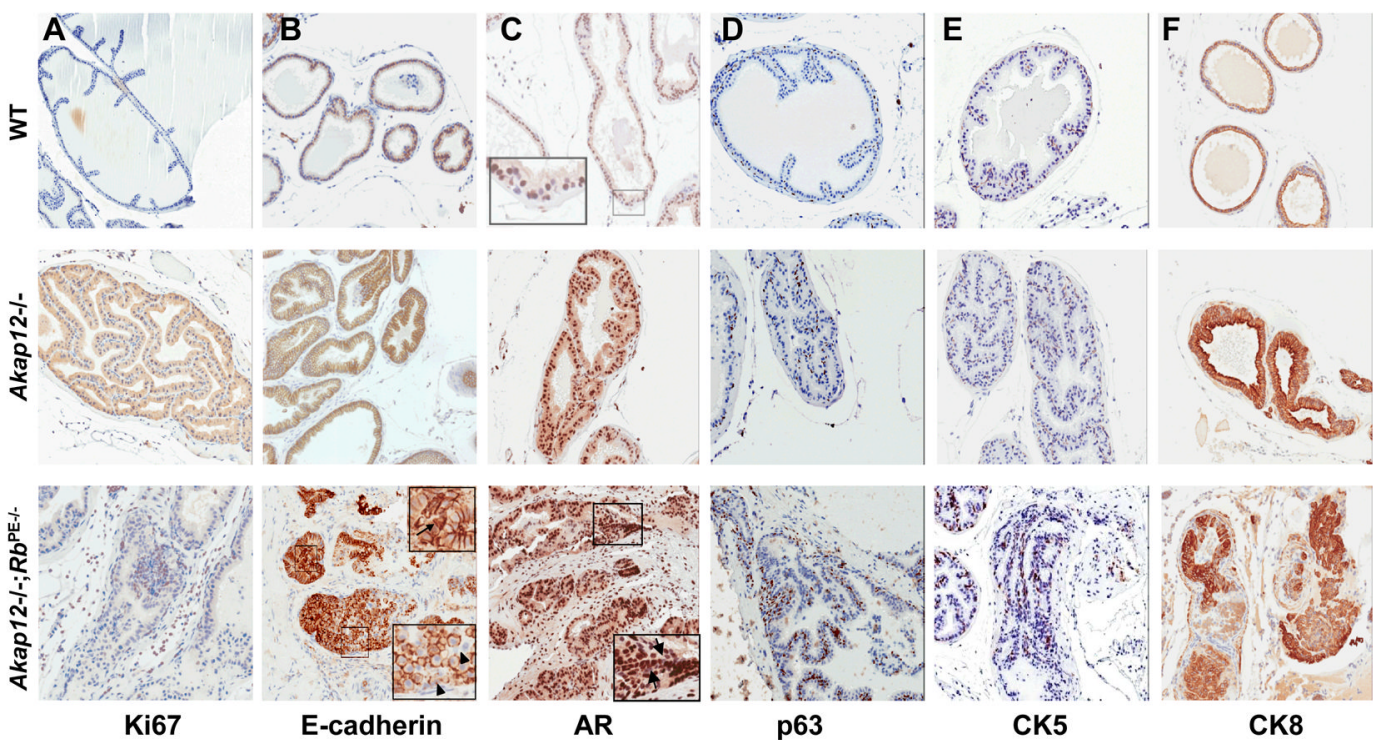

Fig. 2. IHC analysis of Akap12-/-; $R b^{\mathrm{PE}-/-}$ PIN lesions AP lobes from 8 month-old WT, Akap12-/-, and Akap12-/-;R $b^{P E-/-}$ mice were stained for Ki-67 (A), E-cadherin (B), AR (C), p63 (D), CK5 (E) or CK8 (F). Cell-cell junctional Ecadherin staining in Panel B (arrow) is contrasted with cytoplasmic staining (arrowhead). A focus of increased nuclear AR staining in Akap12-/-;Rb $b^{P E-l-}$ vs. WT is shown in Panel C (insets; arrows). 

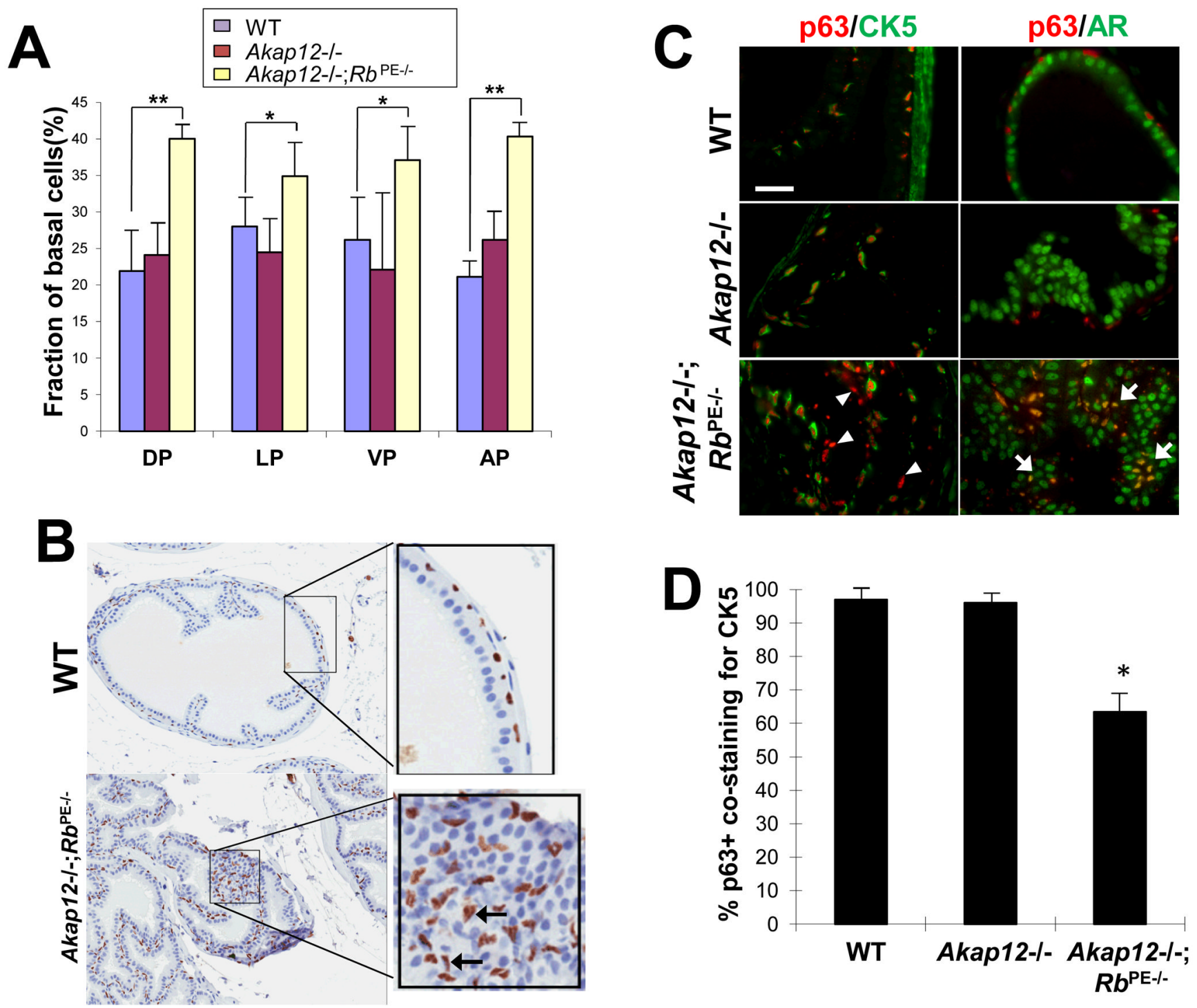

Fig. 3. Akap12-/-; $R b^{P E-/-}$ PIN lesions display hyperplasia of p63-positive, CK5-negative basal epithelial cells

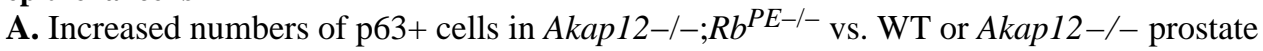
lobes. p63+ cells counted in 6 independent low-magnification fields (at least 40 cells/field) were normalized to total numbers of epithelial cells. Error bars, S.E.; *, p<0.05; **, p<0.01. B. IHC staining for p63 in Akap 12-I-;R $b^{P E-l-}$ vs. WT AP lobes (8 month-old) showing an increase in basal cells displaying a loss of normal basal cell morphology Akap12-I-;R $b^{P E-/-}$ mice. C. IFA co-staining for p63/CK5 or p63/AR in Akap12-l-;R $b^{P E-l-}$ vs. WT or Akap12-l- AP lobes. Arrowheads, $\mathrm{p} 63^{+} \mathrm{CK}^{-}$basal cells in PIN lesions; arrows, $\mathrm{p}^{+} 3^{+} \mathrm{AR}^{+}$ basal cells. Scale bar, $20 \mu \mathrm{m}$. D. Six independent microscopic fields of VP lobes in Panel B co-stained for p63 and CK5 (at least 25 cells/field) were quantified for p63+ cells that costain for CK5. Error bars, S.E.; *, p $<0.01$. 

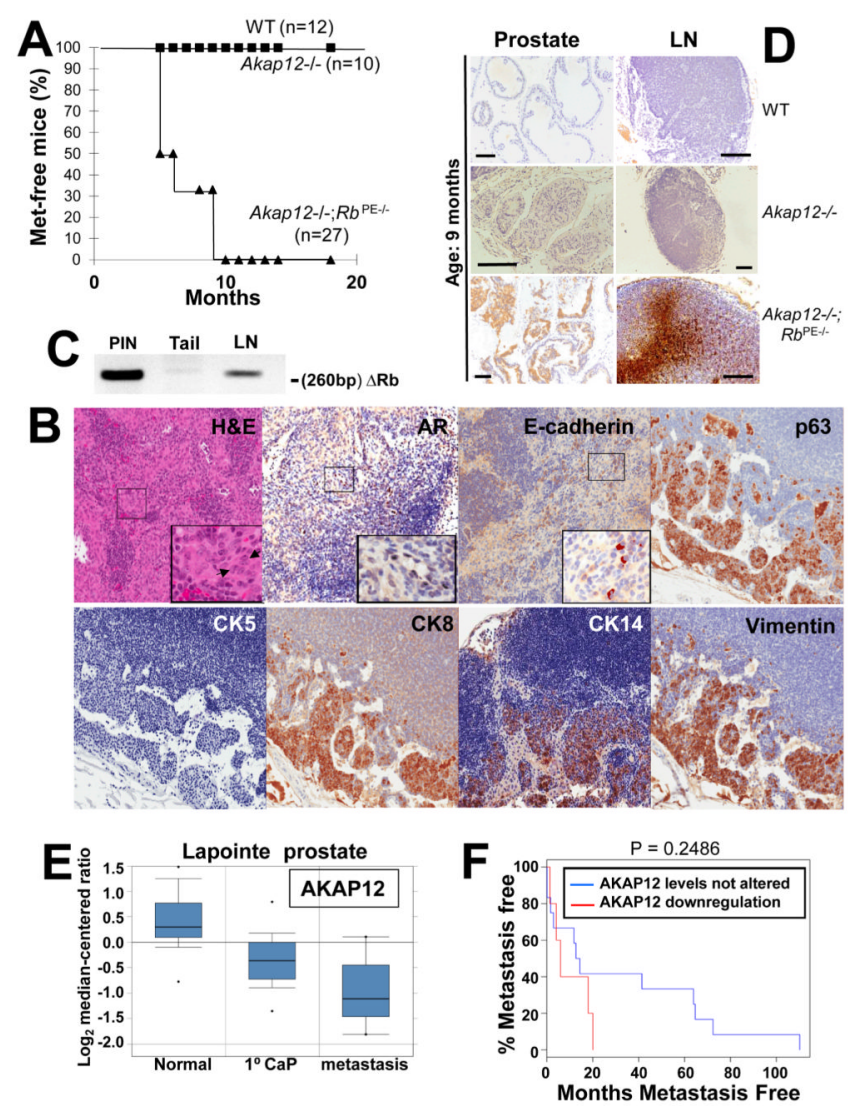

Fig. 4. Lymph node metastases in Akap12-/-; R $\boldsymbol{B}^{P E-/-}$ mice

A. Incidence of LN metastasis in WT, Akap12-I-, and Akap12-/-;Rb ${ }^{P E-/-}$ mice based on numbers of mice (n) per genotype. B. Characterization of cell markers by IHC. Pelvic LNs from 13 month-old Akap12-l-;Rb ${ }^{P E-l-}$ mice were analyzed for basal (p63, CK5 and CK14) or luminal (AR, E-cadherin) epithelial cells markers, as well for a marker of mesenchymal transition (vimentin). Note the lack of CK5 staining. Arrow, examples of HG-PIN-like cells with pleiomorphic nuclei and abnormal nuclear:cytoplasmic ratios. C. PCR-based confirmation of the prostatic origin of the LN tumor. PIN or LN metastasis lesions were isolated using laser capture microscopy (RPCI Pathology Resource Network- Carl Morrison, Director), and DNA isolated using a Qiagen QIAamp DNA Micro Kit was subjected to PCR using primers specific for the deleted $\mathrm{Rb}^{\mathrm{fl} / \mathrm{fl}}$ allele (Rb212M5', Rb18M3'; Supplementary fig. S1A) and then analyzed by agarose gel electrophoresis. DNA isolated from the tail snip

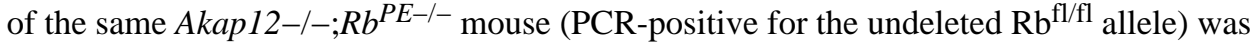
used as a negative control. D. RNA in situ hybridization for Cre expression in primary prostate (DP) vs. LN in 9 month-old WT, Akap 12-/- or Akap12-/-;Rb $b^{\mathrm{PE}-/-}$ mice. Scale bars, $100 \mu \mathrm{m}$. E. Oncomine analysis (http://www.oncomine.org) of Lapointe prostate cancer expression data (39) showing AKAP12 downregulation in human prostate cancer metastasis compared to levels in primary prostate cancer (" $1{ }^{\circ} \mathrm{CaP}$ ") or normal prostate tissue. $\mathbf{F}$. Kaplan-Meier plot analysis (http://www.cbioportal.org/public-portal/) of metastasis occurrence vs. time to onset in 37 prostate cancer metastasis cases from Taylor et al. (38) in which 11 cases (29.7\%) displayed AKAP12 downregulation compared to levels in primary $\mathrm{CaP}$ lesions, versus 26 cases with no change in AKAP12 levels. 


\section{Table 1}

Summary of prostatic epithelial defects in Akap12-I-;Rb $b^{P E-I-}$ mice.

\begin{tabular}{lcccc}
\hline Genotype $^{\boldsymbol{a}}$ & Normal & Hyperplasia & LGPIN & HGPIN \\
\hline Wild-type & $10 / 11(91 \%)$ & $1 / 11(9 \%)$ & $0 / 11$ & $0 / 11$ \\
Akap12-l- & $0 / 8$ & $8 / 8(100 \%)$ & $1 / 8(13 \%)^{b}$ & $0 / 8$ \\
Akap12-I-; $R b^{P E-l-}$ & $0 / 21$ & $21 / 21(100 \%)$ & $20 / 21(95 \%)$ & $6 / 21(29 \%)$ \\
\hline
\end{tabular}

$a_{6-13 \text { months-old; }}$

$b_{18 \text { month-old }}$ 
Table 2

Frequency of LN metastases

\begin{tabular}{|c|c|}
\hline Genotype $^{a}$ & LN metastases $b$ \\
\hline Wild-type & $0 / 11$ \\
\hline Akap12-I- & $0 / 8$ \\
\hline Akap12-l-; $R b^{P E-I-}$ & $15 / 18(83 \%)$ \\
\hline
\end{tabular}

